\title{
Racial-Sex Disparities-A Challenging Battle Against Cancer Mortality in the USA
}

\author{
Wenjiang J. Fu
}

Received: 21 May 2014 / Revised: 12 August 2014 / Accepted: 4 September 2014 / Published online: 2 October 2014

(C) W. Montague Cobb-NMA Health Institute 2014

\begin{abstract}
Decline in US cancer mortality has recently been reported, based on either pooled mortality of all cancer sites or age-adjusted mortality rates of specific sites. While the former could be dominated by a few cancer sites and would not reflect that of other sites, the latter used the US 2000 Population as reference for age-standardization, which was lack of justification. This study aimed to examine US cancer mortality trend and disparities in sites, races, and sex. We studied cancer incidence-based mortality by race and sex from 1974 to 2008 of cervix, prostate, colon and rectum, lung, leukemia, liver, pancreas, and stomach in the Surveillance, Epidemiology, and End Results database. We developed a model-based mortality rate and examined rate ratio of each calendar period to the first period within each race-sex group. Cancer mortality of cervix, colon and rectum, leukemia, and stomach declined in all groups. Prostate cancer increased first in all racial groups and decreased thereafter at different pace. Lung cancer declined among males of all races but increased among females. Liver cancer increased steadily fast among white and black females, doubled in whites and black males, and climbed slowly in other races. Pancreas cancer declined among black males and females, and changed little among others. Cancer mortality trend presents heterogeneity across sites, races, and sex. Recently observed mortality decline may not reflect every cancer site or group. More effort needs to focus on specific race-sex groups that had increasing lung and liver cancer mortality.
\end{abstract}

Electronic supplementary material The online version of this article (doi:10.1007/s40615-014-0059-6) contains supplementary material, which is available to authorized users.

W. J. Fu $(\bowtie)$

Department of Epidemiology and Biostatistics, Michigan State

University, 909 Fee Road, West Fee Hall, Suite B601, East Lansing,

MI 48824, USA

e-mail: fuw@msu.edu
Keywords Cancer mortality $\cdot$ Incidence $\cdot$ Disparities $\cdot$ Rate ratio $\cdot$ Trend

\section{Introduction}

Cancer mortality remains one of the leading causes of death in the USA though a decline has been reported in recent years [1-4]. It is essential to examine temporal trend of incidence or mortality rate in evaluating cancer intervention programs, such as smoking cessation or dietary intervention, and a successful program is expected to lower disease incidence through primary prevention or disease mortality through early diagnosis and early treatment [5-7]. A decline in cancer mortality was observed in a recent age-period-cohort (APC) study of pooled mortality rate of all cancer sites [4]. A similar decreasing trend of most cancer sites was also reported in a Surveillance, Epidemiology, and End Results (SEER) Cancer Statistics Review 1975-2010 [3] by examining age-adjusted mortality rate using the US 2000 Population as reference [8]. Given such celebrating achievement in fighting against cancer mortality, one may wonder: Has the mortality been declining in all primary cancer sites? Or has it been declining across all race-sex groups?

An examination of the temporal trend of cancer mortality rate may provide a clue to the above questions and may guide future cancer intervention programs. Since the pooled mortality of all cancer sites could be dominated by a few sites of large numbers of deaths, a decline in cancer mortality rate of these sites would not reflect the trend of others. In addition, concerns have been raised about estimating mortality trend with age-adjusted rate using the US 2000 Population as reference [9-13]. It was reported that age-adjusted rate varied largely with the reference population in direct age-standardization. The use of the US 2000 Population was questioned by a World Health Organization (WHO) report because "there is 
clearly no conceptual justification for choosing one standard over another, hence the choice is arbitrary" [10]. Furthermore, a decline in racial/ethnic and socioeconomic disparities was attributed to a "statistical illusion" caused by the change of reference population from the USA year 1940 population, 1970 population, to 2000 population in age-standardization [11]. A recent study further found that the age-standardization procedure using the US 2000 Population as reference overestimates prostate cancer mortality rate by up to $91 \%$ and underestimates cancer fatality rate by up to $78 \%$ [14]. Given the above concerns, studies of temporal trend of cancer mortality need to use caution, and more studies using alternative approaches are needed to (1) further examine the recent decline in cancer mortality by site, (2) identify cancers from which mortality did not decline or even increased, and (3) study disparities in cancer mortality among race-sex groups and identify those who suffered the most [11].

To examine the cancer mortality trend and to assess the disparities in race and sex, we analyzed cancer incidencebased mortality (IBM) from 1974 to 2008. Specifically, we studied the IBM rates from 1984 to 2008 of eight cancer sites (cervix, prostate, lung, colon and rectum, leukemia, liver, pancreas, and stomach) from the Surveillance, Epidemiology, and End Results (SEER) cancer registry database [1]. We developed a model-based mortality rate and rate ratio to examine the temporal trend within each race-sex group for a given cancer site. Our method allowed the examination of temporal trend within each race-sex group and identification of disparities across race-sex groups. We further compared the mortality trend with the report in the SEER Cancer Statistics Review and identified similarities and differences, which may provide insights to the disparities in cancer mortality.

\section{Methods}

Data

The cancer mortality data were obtained from the SEER database with the SEER*Stat software using the IncidenceBased Mortality SEER 9 Registrations Research Data, November 2010 submission (1973-2008) with Katrina / Rita Population Adjustment [15]. The SEER program, initiated by the National Cancer Institute in 1973, aims to provide information about cancer statistics to help to reduce the burden of cancer patients. The program started with nine states and metropolitan areas in the USA (or the SEER 9), including the state of Connecticut, Iowa, New Mexico, Utah, and Hawaii and the metropolitan areas of Detroit, San-FranciscoOakland, Atlanta, and the Seattle-Puget Sound. It expanded in recent years to 13 states and metropolitan areas in the USA, including areas with minorities and American Indians.
Compared to the US population, the SEER population has similar characteristics, including proportion of foreign born individuals, proportion below poverty level, and high school educational level. For this reason, findings of cancer incidence and mortality rates from the SEER program have been regarded as the findings in the USA. More detailed information about the SEER program and cancer statistics can be found on the website of the SEER program [16]. We use SEER 9 data for the mortality trend estimation for its relatively long duration of cancer registry to allow the examination of the cancer mortality trend. It has individual patient demographic information and clinical information, including age, sex, race, cancer site, year of diagnosis and death year, and so on.

For each cancer site, a specific race (white, black, and other races), and sex (male and female) group, we calculated the IBM rate using a 10-year window (requiring disease exposure of 10 years or longer before death) based on the death records of cancer patients in the SEER database [17, 18], following the instruction of calculating 10-year IBM rate [19]. Both the number of deaths and the person-year exposure were generated for each age-period-specific group of 5-year interval for a given cancer site, race, and sex in the periods 1984-1988 to 2004-2008. The procedure was repeated for different race-sex groups and further repeated for different cancer sites.

We selected prostate cancer and other seven sites because the 5-year relative survival of the other seven sites was much smaller than that of prostate cancer (Table 1 in online Appendix) [3], for which a method of IBM rate was developed and validated based on a window of 10 -year exposure [17, $18]$. Deaths from cancers with a smaller 5-year relative survival were more likely to be counted within a 10 -year window of exposure for accurate estimation of cancer cases and exposure. We confirmed this through a comparison between 10and 8-year IBM data of liver cancer, showing no major difference in the number of cases and person-year exposure (data not shown). For the same reason, we kept prostate cancer in this study, but excluded female breast cancer because of its relatively large 5-year survival rate.

We further generated age-period-specific incidence rate and corresponding population for each race-sex group from 1974-1978 to 2004-2008 using the SEER 9 database. The incidence had two extra periods, 1974-1978 and 1979-1983, compared to the IBM rate because the IBM was restricted to deaths in the SEER program and was calculated after the initial 10 years from the start of the SEER program in 1973.

\section{Statistical Analysis}

To examine temporal trend of the IBM rate, we calculated a model-based mortality rate (MMR) of each 5-year calendar period (from 1984-1988 to 2004-2008) and a rate ratio (RR) to the first period (1984-1988) of each cancer site. The MMR 
and the RR were defined based on the APC classification model (1) below fitted to the IBM data, for example, lung cancer mortality rate and number of deaths among black females (Table 2 in online Appendix). The APC model studies disease rate displayed in an $a \times p$ table (e.g., Table 2 in online Appendix) with $a$ rows of age groups and $p$ columns of periods, aiming to estimate age effects $\alpha_{1}, \ldots, \alpha_{a}$ of $a$ rows, period effects $\beta_{1}, \ldots, \beta_{p}$ of $p$ columns, and cohort effects $\gamma_{1}, \ldots, \gamma_{a+p-1}$ of $(a+p-1)$ diagonals [20].

$\log \left(E_{i j}\right)=\log \left(n_{i j}\right)+\mu+\alpha_{i}+\beta_{j}+\gamma_{a-i+j}$

where $E_{i j}$ is the expected number of deaths in cell $(i, j)$ and $n_{i j}$ is the population exposure in the same cell. $\mu$ is the model intercept.

We define the MMR and RR below using fitted values of model (1) because the fitted values are uniquely determined despite the identifiability problem of the APC model [20,21], for which a unique set of model parameters is difficult to determine [20-23]. The model parameters $\alpha_{i}, \beta_{j}, \gamma_{a-i+j}$ can be estimated using Poisson regression with a constraint on the parameters, such as $\alpha_{1}=\alpha_{2}$. The model-based definitions of MMR and RR filter the random noise in the mortality rate data while taking the variability in age, period, and cohort into consideration.

$M M R_{i j}=\exp \left(\mu+\alpha_{i}+\beta_{j}+\gamma_{a-i+j}\right), i=1, \ldots a, j=1, \ldots p$

$$
\begin{aligned}
R R_{j} & =\frac{\sum_{i=1}^{a}\left(n_{i o} M M R_{i j}\right)}{\sum_{i=1}^{a}\left(n_{i o} M M R_{i 1}\right)} \\
& =\exp \left(\beta_{j}-\beta_{1}\right) \frac{\sum_{i=1}^{a}\left(n_{i o} \exp \left(\alpha_{i}+\gamma_{a-i+j}\right)\right)}{\sum_{i=1}^{a}\left(n_{i o} \exp \left(\alpha_{i}+\gamma_{a-i+1}\right)\right)}, \\
j & =1, \ldots p
\end{aligned}
$$

where $n_{i o}$ is a reference population for the agestandardization procedure. In this study, we chose the population structure of each race-sex group in period 1984-1988 as the reference for that group because the population structure remains relatively stable within each race-sex group but may vary largely across groups, as shown in Fig. 1 in the online Appendix. To examine the temporal trend of mortality, we plot the RR against the period by race-sex group of each disease.

The population proportion in the first period of each racesex group is the preferred reference to the US 2000 population since the latter may not represent accurately the population structure in each group with a large discrepancy in age profile as shown in Fig. 1 in the online Appendix, leading to large deviation in age-adjusted MMR and RR.
To examine the temporal trend of cancer incidence, we calculated the model-based incidence rate (MIR), similar to the MMR, and its RR using the MIR.

$$
\begin{aligned}
R R_{j} & =\frac{\sum_{i=1}^{a}\left(n_{i o} M I R_{i j}\right)}{\sum_{i=1}^{a}\left(n_{i o} M I R_{i 3}\right)} \\
& =\exp \left(\beta_{j}-\beta_{3}\right) \frac{\sum_{i=1}^{a}\left(n_{i o} \exp \left(\alpha_{i}+\gamma_{a-i+j}\right)\right)}{\sum_{i=1}^{a}\left(n_{i o} \exp \left(\alpha_{i}+\gamma_{a-i+3}\right)\right)}, \\
j & =1, \ldots p, p=7
\end{aligned}
$$

To compare with the mortality trend, we use the same reference period (1984-1988 corresponding to $j=3$ ) for the $\mathrm{RR}$, plot the RR within each race-sex group, and examine them between incidence and mortality for each race-sex group. The extended trend of the RR in periods 1974-78 and 1979-1983 provides extra information of incidence trend.

\section{Results}

\section{Mortality Trend}

Figures 1 and 2 present temporal trend of mortality of eight cancer sites by race-sex from 1984 to 2008. Cancer mortality of the cervix, leukemia, colon and rectum, and stomach declined consistently in all racial or race-sex groups. Specifically, cancer of the cervix decreased around $40-50 \%$, leukemia declined 20-30\%, and cancer of the stomach dropped 30-40\%. While cancer of the colon and rectum declined 10-20\% among black males and females and females of other races, it dropped 30-40\% among white males and females and males of other races. Prostate cancer increased first in all racial groups and decreased thereafter, resulting in a $20 \%$ decline among the whites and little change in others. Lung cancer declined by $30 \%$ among the white and black males, and by $10 \%$ among males of other races, but increased by more than $10 \%$ among females of all races. Liver cancer increased more than doubled among white and black males, increased steadily fast by $60-80 \%$ among white and black females, and increased by $30 \%$ among other races. Pancreas cancer declined by $10 \%$ among black males and females and little changed among the whites and other races.

Fluctuation was observed in leukemia mortality among males and females of other races. Fluctuation was also observed in pancreas cancer mortality among females of other races. Both black males and females showed a slow decline in pancreas cancer mortality with fluctuation. 
Fig. 1 Model-based RR of mortality (1984-2008) by race and sex of eight cancer sites based on the IBM rate from SEER registry adjusted with the age structure of the first period (1984 1988) person-year exposure of each race-sex subgroup. Race: black curve - white; red curveblack; green curve - other race. Sex: solid curve-female; dashed curve-male a
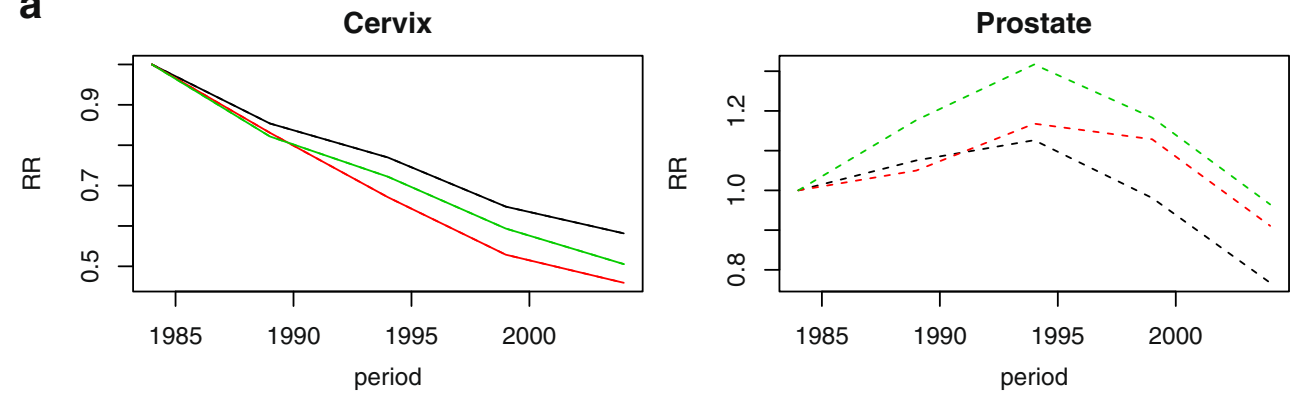

Colon+Rectum

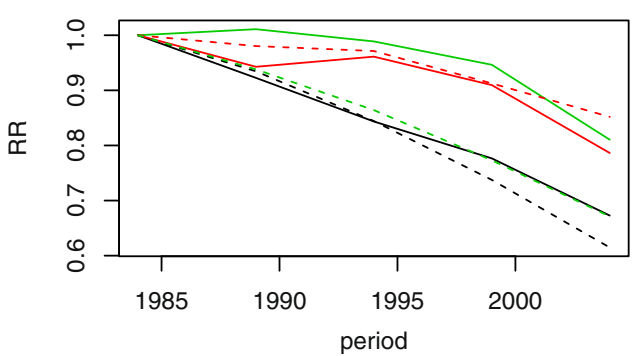

b

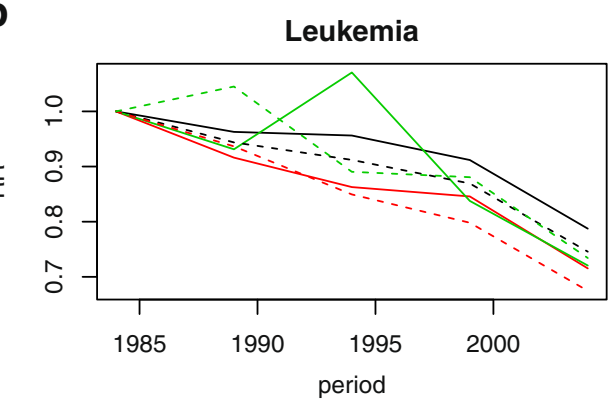

Pancreas

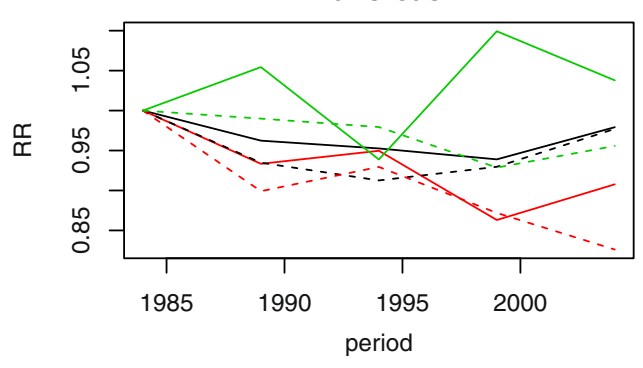

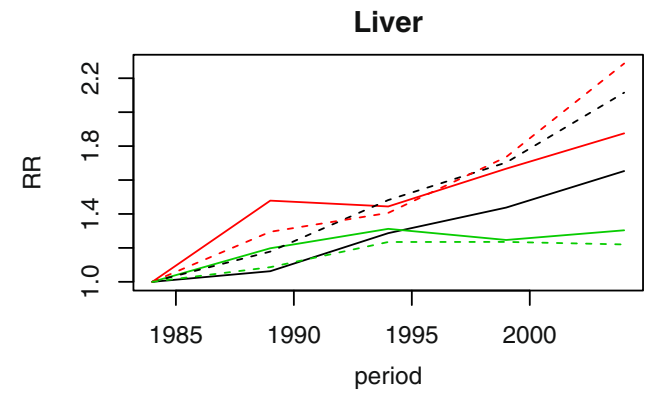

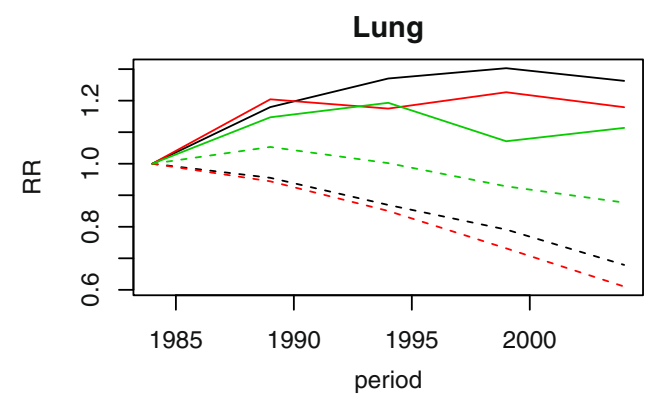

Liver

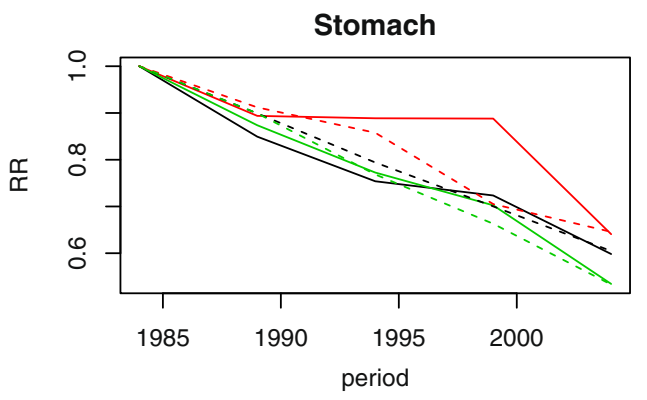

Incidence Trend

To understand the changes in mortality trend and heterogeneity across race-sex groups, we further examined incidence trend from 1974-1978 to 2004-2008 and compared it with the mortality. The incidence presented a steady decline in cancers of the cervix and stomach in all groups, consistent with the decline in mortality. The increasing incidence of liver cancer in all groups was also consistent with the increasing mortality. The decreasing lung cancer incidence among white and black males was consistent with the decreasing mortality, while the increasing incidence among females was consistent with the increasing mortality. The slightly increasing then decreasing lung cancer incidence among males of other races was consistent with the slightly decreasing mortality. The decreasing incidence of colon and rectum cancer was consistent with the fast decreasing mortality among white males and females and males of other races, while the slowly decreasing mortality among black males and females and females of other races could be attributed to the flattened incidence with fluctuations. The steadily fast decrease of leukemia mortality in all groups could well be attributed to the advances in treatment 
Fig. 2 Model-based RR of incidence (1974-2008) by race and sex of eight cancer sites based on the IBM rate from SEER registry adjusted with the age structure of the third period (1984-1988) person-year exposure of each race-sex subgroup. Race: black curve - white; red curve-black; green curve- other race. Sex: solid curve - female; dashed curve - male a
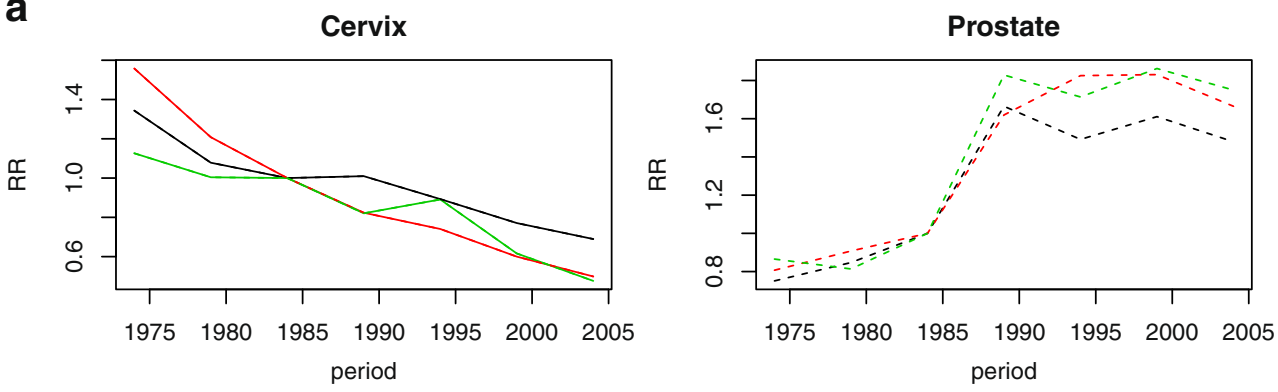

Colon+Rectum
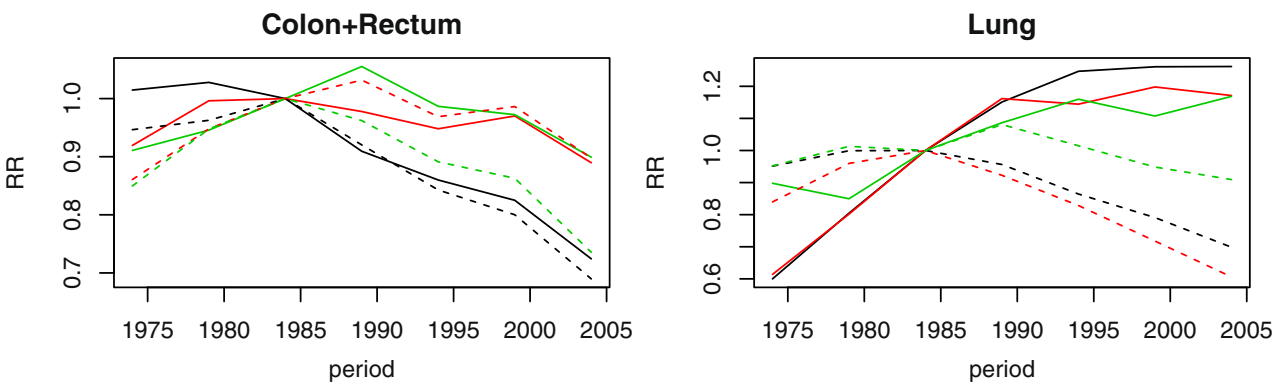

b
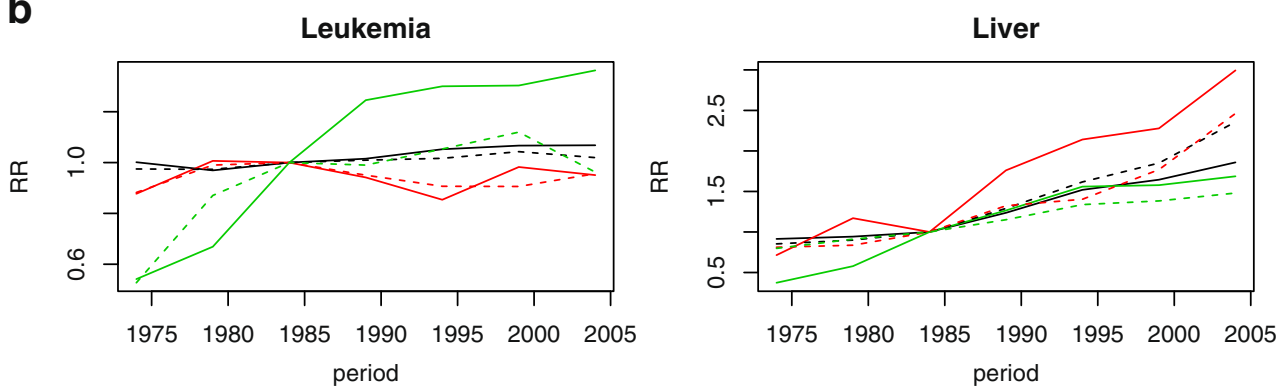

Pancreas

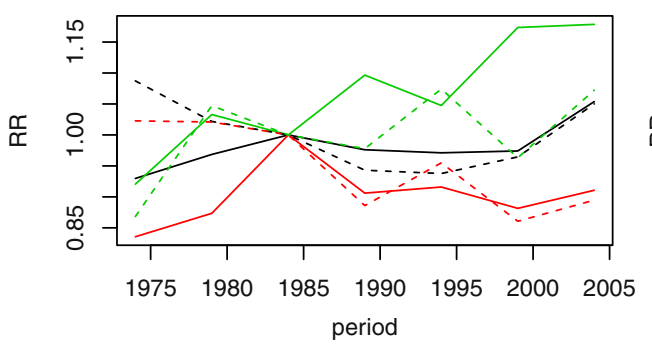

Stomach

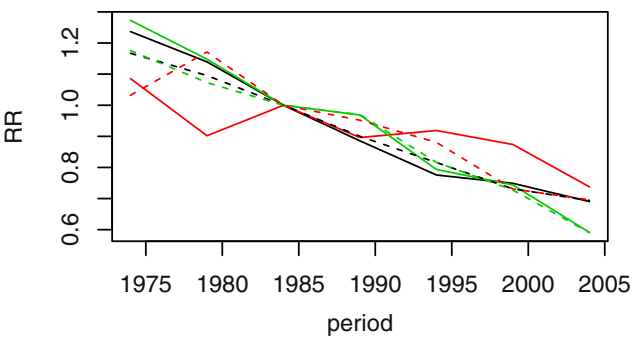

because the incidence was flat in all groups except for an increasing trend among females of other races. Prostate cancer presented a sharp increase in incidence from 1974-1978 to 1988-1993 followed by a level off in all groups. This could partly explain the initial increase in mortality in all groups from 1984-1988 to 1994-1998, followed by a decline thereafter when the incidence leveled off. Pancreas cancer incidence fluctuated in all groups, which was also consistent with the fluctuation in mortality.

It can be concluded in general that increasing mortality was highly correlated with increasing incidence (e.g., liver cancer, prostate cancer, and female lung cancer) except for leukemia, for which mortality decreased while incidence did not. Fast decreasing mortality was also highly correlated with decreasing incidence (e.g., cancer of the cervix, stomach, and colon and rectum among whites).

\section{Discussion}

War against Cancer

The war against cancer has been progressing rapidly with the advancement in medical research and technologies that have made numerous promising treatments and therapeutics 
available. Despite the successes in treatment and therapeutics, the battle against cancer has been difficult, thanks to the disparities among racial groups, sex and socioeconomic classes. Strategic plans are needed to win the war against cancer, with special emphasis on studies of the causes, prevention programs based on cellular, molecular and genomic research evidence, and global surveillance, public health policy, and equity of cancer [24-26]. Cumulative evidence has shown that studies of the war against cancer without emphasizing the disparities would be incomplete [27-29].

\section{Heterogeneity in Mortality Trend}

Racial, sex, and socioeconomic disparities have been studied extensively in the literature and still remain a major challenge to public health [10,27-29]. Our findings in temporal trend of cancer mortality rate of eight cancers over the past 30 years provide a strong evidence of racial and sex disparities, and further demonstrate that although decline in cancer mortality has been reported in recent years, it may not reflect the trend in every cancer site. For example, liver cancer presented an increasing trend, varying largely across race-sex groups. Lung cancer mortality decreased among males but increased among females, consistently across all racial groups. Furthermore, the disparities in lung cancer incidence and mortality between males and females indicate that cancer intervention programs need to address disparities and target on high-risk populations. In addition, the increasing incidence and mortality in prostate cancer and liver cancer and the fluctuating pattern in incidence and mortality of pancreas cancer indicate the needs of both efficient intervention programs and effective treatments and therapeutics.

\section{Impact of Incidence and Mortality Trend}

Although cancer mortality in the USA has been reported to decline, the prediction of worldwide skyrocketing cancer incidence and mortality in the next 20 years by the World Cancer Report 2014 presents a sharp turn of cancer mortality trend [30], prompting a reexamination of cancer mortality. Specifically, the reported decline of cancer mortality of all caner sites combined may not reflect the trend of some specific cancer sites (such as liver cancer shown in Fig. 1) as it may be dominated by a few large cancer sites with declining mortality. Further, it has recently been found that the direct age-standardization method may overestimate cancer mortality rate when the age structure of a standard healthy population is adopted in the age-standardization. These methodological limitations in previous approaches and the severe consequences of inaccurate estimation of cancer mortality make it urgent to further examine the mortality trend using different analytic methods. Our newly developed model-based mortality rate and rate ratio approach further confirmed most findings in the SEER Cancer Statistics Review [3]. Specifically, it confirmed the declining mortality of cervix and stomach cancer among all groups, lung cancer among males of all racial groups, and colon and rectum cancer among white males and females. It also confirmed the increasing mortality of liver cancer of all race-sex groups and lung cancer among females of all racial groups.

In addition, we also observed differences between our findings and those in the SEER Cancer Statistics Review. The latter reported that leukemia mortality increased from 1975 to early 1990s with a peak around 1992 among both black males and females, but steadily decreased among both white males and females ([3] Fig. 13.1). In contrast, our analysis observed a steadily decreasing mortality among both black and white males and females with a faster decline among the blacks than the whites. To gain insight of such discrepancy, we examined leukemia incidence trend for possible explanation. The SEER Cancer Statistics Review reported increasing incidence of leukemia among both white males and females but rather a flattened incidence among both black males and females, while our analysis observed a similar increasing trend among both white males and females since 1984 but a decreasing trend in both black males and females. If the observation in both reports that an increasing incidence trend among the whites was followed by a steadily decreasing mortality trend was true, it would imply, assuming equal efficacy of treatment among the whites and blacks, that leukemia mortality would have declined faster among the blacks due to the slower increase in incidence than the whites, which was consistent with our finding. However, this makes it difficult to explain the observations in the SEER Cancer Statistics Review that a flattened incidence (virtually no increase) among the blacks was followed by an increasing-thenslightly-decreasing mortality trend. Such a finding, if it was true, would strongly indicate racial disparities in leukemia mortality and could be explained potentially by the low socioeconomic status and limited access to healthcare among the blacks, indicating strong racial/socioeconomic disparities between the blacks and the whites [31-33]. Further investigation linking mortality data of leukemia patients to the socioeconomic status is needed to test this hypothesis, which is out of the scope of this study and will be reported elsewhere.

Compared to the previous study of pooled cancer mortality, our findings not only identified cancer sites that presented increasing mortality, but also identified populations who had increasing cancer mortality. The identification of these populations of increasing risk is also supported and confirmed by increasing incidence and may provide guidance to future cancer programs.

Racial/sex disparities in cancer mortality have also been reported in the literature to analyze individual patient data adjusting for a number of risk factors, including age, disease 
stage, genetic mutation, treatment received, and socioeconomic status (SES) or employment status. A study of female breast cancer mortality rate identified higher hazard rate among black women than white women adjusting for estrogen receptor expression level [34]. The gap between higher breast cancer mortality rate of black women and lower mortality rate of white women was shown to grow continually nationwide and in the metropolitan areas with large black population, such as Chicago [35]. No major racial differences in the efficacy of breast cancer treatment have been concluded in the literature between white and black women [36]. A higher hazard ratio of cervical cancer mortality among African American women was also found than white women [37]. Reasons for the disparities of cervical cancer mortality are likely multifactorial, including early screening, treatment procedures, SES, and so on after adjusting for common risk factors (e.g., age, marital status, stage of diseases, treatment, grade and histology, etc.) [36, 37]. Racial disparities were also found between black and white Americans in colorectal cancer mortality $[38,39]$ and in the treatment procedures of colorectal cancer [36]. Racial disparities in lung cancer mortality and nonsmall cell lung cancer treatment were found between white and black Americans but the gap is narrowing down over time [40, 41]. No statistical significant differences were found in tumor response or disease progression [36]. Sex disparities were also found in lung cancer survival with women having a better chance to survive [42]. Racial disparities were also found in very low-risk prostate cancer patients between African American and the whites [43], while race may seem to be an independent factor for the prognosis, African American men are less likely than the whites to receive expensive treatment or more innovative treatments [36]. Liver cancer mortality presents an increasing trend by race and sex, and the racial disparities were observed in a long-term study from 1969 to 2011, even after being stratified by county unemployment level [44]. While leukemia mortality has been found to be partly attributable to certain genetic factors, racial disparities play an important role in the mortality [30]. The racial disparities were reported to gradually vanish in stomach cancer mortality [45].

While it is not straightforward to compare our findings with the above disparity studies because of the different racial groups studied and different periods of time examined, a comparison of our findings in racial-sex disparities reveals interesting observations. Our analysis shows that liver cancer mortality increased in all racial-sex groups, it increased at a faster pace among white and black males than white and black females (Fig. 1). While an increase was reported among the blacks with higher mortality than the whites, it was at a relatively slower pace among the blacks than the whites [44]. Our analysis shows that lung cancer mortality rates increased among females in all racial groups while it declined among males in all racial groups. This result can be largely explained by the observations that smoking and consequently lung cancer incidence rate decreased more rapidly among males than females [46], but it differs largely from the report that women had a better chance to survive lung cancer [42]. These differences prompt further investigations of cancer mortality rates, preferably using more advanced statistical methods to address methodological issues.

Given the long period of time covered in this study, the cancer mortality trend may partly reflect the advances of cancer treatment, medical programs for screening, early detection, and early treatment. For example, the increasingthen-decreasing mortality of prostate cancer may reflect the increasing-then-flat incidence trend, which might be affected largely by the prostate cancer screening programs, such as the use of prostate-specific antigen (PSA) for screening, before the year 2000 and so on. The big contrast in lung cancer mortality between males and females may partly due to the behavior of increased female smokers and relatively less increased or decreased male smokers [47].

\section{Conclusion}

The steadily increasing incidence and mortality trend of liver cancer and female lung cancer poses great challenges in the battle against cancer mortality. Although lung cancer incidence and mortality have declined among males of all racial groups in recent years, thanks to cancer intervention programs (e.g. smoking cessation), these programs have yet been as effective among females. Meanwhile, liver cancer mortality has more than doubled in black and white males, and increased by more than $50 \%$ among black and white females. More strategic plans combining prevention, therapeutics, and treatment are required in the battle against cancer mortality. Implementation of such programs can also be challenging and may require joint effort in epidemiology, public health, nutrition, social, and behavior sciences [24-26].

It is anticipated that advances in biomedical research will lead to more effective medical treatment and therapeutics. However, the racial, sex, and socioeconomic disparities in the USA may unlikely disappear in a short period of time during the long-term fight against cancer. With the globalization of economy and migration of world population, racial-sex disparities will soon become a major public health task in many countries, posing greater challenges to the battle against cancer mortality. One can be sure that although the fighting against cancer mortality has been more promising than ever before, it still remains an uphill battle with various challenges ahead. 
Acknowledgments This work was partly supported by the National Institutes of Health/National Cancer Institute Grants CA83010 and CA90301.

Conflict of Interest Author Wenjiang Fu declares that he has no conflict of interest.

Animal or Human Studies No animal or human studies were carried out by the author for this article.

\section{References}

1. American Cancer Society, SEER Cancer Stat Fact Sheet, http://seer. cancer.gov/statfacts/. Accessed 26 Oct 2013.

2. American Cancer Society. Cancer Facts and Figs. 2012, Atlanta, GA. 2012.http://www.cancer.org/acs/groups/content/@ epidemiologysurveilance/documents/ document/acspc-031941.pdf. Accessed 26 Oct 2013.

3. Howlader N, Noone AM, Krapcho M, et al. (eds). SEER Cancer Statistics Review, 1975-2010, National Cancer Institute. Bethesda, MD. http://seer.cancer.gov/csr/1975_2010/. Accessed 26 Oct 2013.

4. Kort EJ, Paneth N, Vande Woode GF. The decline in the US cancer mortality rate in people born since 1925. Canc Res. 2009;69:6500-5.

5. World Cancer Research Fund. American Institute for Cancer Research: Food, Nutrition and the Prevention of Cancer: A Global Perspective. Washington, DC: The Institute, 1997. http://www. dietandcancerreport.org/. Accessed 26 Oct 2013.

6. U.S. Department of Health and Human Services.: The Health Consequences of Involuntary Exposure to Tobacco Smoke: A Report of the Surgeon General. Atlanta, GA: U.S. Department of Health and Human Services, Centers for Disease Control and Prevention, Coordinating Center for Health Promotion, National Center for Chronic Disease Prevention and Health Promotion, Office on Smoking and Health. 2006. http://www.surgeongeneral. gov/library/reports/secondhandsmoke/fullreport.pdf. Accessed 26 Oct 2013.

7. Gail MH, Costantino JP, Bryant J, et al. Weighing the risks and benefits of tamoxifen treatment for preventing breast cancer. J Nat Can Inst. 1999;91:1829-46.

8. Sorlie PD, Thom AJ, Manolio T. Age-adjusted death rates: Consequences of the year 2000 standard. Ann Epi. 1999;9:93-100.

9. Ahmad OB, Boschi-Pinto AD. Lopez, et al. Age standardization of rates: a new WHO standard. (GPE Discussion Paper Series: No. 31 EIP/GPE/EBD World Health Organization 2001). http://www.who. int/healthinfo/paper31.pdf. Access 26 Oct 2013.

10. Krieger N, Williams DR. Changing to the 2000 standard million: are declining racial/ethnic and socioeconomic inequalities in health real progress or statistical illusion? Am J Pub Heal. 2001;91:1209-13.

11. Pamuk ER. Cautiously adjusting to the new millennium: Changing to the 2000 population standard. Am J Pub Heal. 2001;91:1174-6.

12. Robson B, Purdie G, Cram F, Simmonds S. Age standardization - an indigenous standard? BMC Emer Them Epi. 2007;4:3. doi:10.1186/ 1742-7622-4-3.

13. Anderson R, Rosenberg HM. Age standardization of death rates: Implementation of the year 2000 standard. Nat Vit Stat Rep. 1998;47:3.

14. Fu M, Todem D, Fu WJ, Ma S. A millennium bug still bites public health - an illustration using cancer mortality. http://arxiv.org/abs/ 1401.7686. Accessed 2 Mar 2014.

15. Chu K, Miller BA, Feuer EJ, Hankey BF. A method for partitioning cancer mortality trends by factors associated with diagnosis: an application to female breast cancer. J Clin Epi. 1994;47:1451-61.
16. National Cancer Institute, SEER program: http://seer.cancer.gov/ registries/. Accessed 10 Aug 2014.

17. Feuer EJ, Merrill RM, Hankey BF. Cancer surveillance series: interpreting trends in prostate cancer-part II: Cause of death misclassification and the recent rise and fall in prostate cancer mortality. $\mathrm{J}$ Nat Can Inst. 1999;91:1025-32.

18. SEER*Stats Software. http://seer.cancer.gov/seerstat/. Accessed 1 Oct 2013

19. National Cancer Institute. Incidence-based mortality sample analysis. http://surveillance.cancer.gov/documents/statistics/ibm/prostate.ibm. demo.pdf. Accessed 26 Oct 2013.

20. Kupper LL, Janis JM, Karmous A, Greenberg BG. Statistical ageperiod-cohort analysis: a review and critique. J Chron Dis. 1985;38: 811-30.

21. Clayton D, Schifflers E. Models for temporal variation in cancer rates. II: age-period-cohort models. Stat Med. 1987;6:469-81.

22. Heuer $\mathrm{C}$. Modeling of time trends and interactions in vital rates using restricted regression splines. Biometrics. 1997;53:161-77.

23. Robertson C, Boyle P. Age-period-cohort analysis of chronic disease rates. I: modelling approach. Stat Med. 1998;17:1305-23.

24. Vinnakota S, Lam NSN. Socioeconomic inequality of cancer mortality in the United States: a spatial data mining approach, Int J Heal Geog 2006; 5:doi:10.1186/1476-072X-5-9.

25. Krieger N, Chen JT, Waterman PD, et al. Geocoding and monitoring of US socioeconomic inequalities in mortality and cancer incidence: does the choice of area-based measure and geographic level matter? the public health disparities geocoding project. Am J Epi. 2002;156: 471-82.

26. Menvielle G, Rey G, Jougla E, Luce D. Diverging trends in educational inequalities in cancer mortality between men and women in the 2000s in France. BMC Pub Heal. 2013;13:823. doi:10.1186/14712458-13-823.

27. Vineis P, Wild CP. Global cancer patterns: Causes and prevention. Lancet. 2013;383(9916):549-57.

28. Hanahan D. Rethinking the war on cancer. Lancet. 2013;383(9916): 558-63.

29. Coleman MP. Cancer survival: Global surveillance will stimulate health policy and improve equity. Lancet. 2013;383(9916):564-73.

30. Stewart BW, Wild CP. World Cancer Report 2014, International Agency of Research on Cancer, http://www.iarc.fr/en/publications/ books/wcr/index.php. Accessed 1 Mar 2014.

31. Patel MI, Ma Y, Mitchell BS, Fhoads KF. Understanding disparities in leukemia: a national study. Can Caus Cont. 2012;23:1831-7.

32. Flowers CR, Pro B. Racial differences in chronic lymphocytic leukemia. Cancer. 2013;119:3593-5.

33. Falchi L, Keating MJ, Wang X, et al. Clinical characteristics, response to therapy and survival of African American patients diagnosed with chronic lymphocytic leukemia: Joint experience of MD anderson cancer center and Duke University medical center. Cancer. 2013;119:3177-85.

34. Menashe I, Anderson WF, Jatoi I, Rosenberg PS. Underlying causes of the black-white racial disparity in breast cancer mortality: a population-based analysis. J Nat Canc Inst. 2009;101:993-1000.

35. Whitman S, Ansell D, Orsi J, Francois T. The racial disparity in breast cancer mortality. J Comm Heal. 2011;36(4):588-96.

36. Shavers VL, Brown ML. Racial and ethnic disparities in the receipt of cancer treatment. J Nat Canc Inst. 2002;94:334-57.

37. Rauh-Hain AJ, Clemmer JT, Bradford LS, Clark RM, Growdon WB, Goodman A, et al. Racial disparities in cervical cancer survival over time. Cancer. 2013;119:3644-52.

38. Robbins AS, Siegel RL, Jemal A. Racial disparities in stage-specific colorectal cancer mortality rates from 1985 to 2008. J Clin Oncol. 2012;30:401-5.

39. Grubbs SS, Polite BN, Carney J, Bowser W, Rogers J, Katurakes N, et al. Eliminating racial disparities in colorectal cancer in the real world: it took a village. J Clin Onc. 2013;31(16):1928-30. 
40. Hardy D, Liu CC, Xia R, Cormier JN, Chan W, White A, et al. Racial disparities and treatment trends in a large cohort of elderly black and white patients with nonsmall cell lung cancer. Cancer. 2009;115: 2199-211.

41. Jemal A, Center MM, Ward E. The convergence of lung cancer rates between blacks and whites under the age of 40. US Canc Epid Biom Prev. 2009;18:3349-52.

42. Wisnivesky JP, Halm EA. Sex differences in lung cancer survival: do tumors behave differently in elderly women? J. Clin Oncol. 25:1705-12

43. Sundi D, Ross AWE, Humphreys EB, Han M, Partin AL, Carter HB, et al. African American men with very low-risk prostate cancer exhibit adverse oncological outcomes after radical prostatectomy: should active surveillance still be an option for them? J Clin Oncol. 2013;31:2991-7.
44. Singh GK, Siahpush M, Altekruse SF. Time trends in liver cancer mortality, incidence, and risk factors by unemployment level and race/ethnic, United States, 1969-2011. J Commun Heal. 2013;38: 926-40.

45. Luyimbazi D, Nelson RA, Chen SL, Kim J. Conditional survival in gastric cancer: does race influence prognosis in long-term survivors? J Clin Oncol. 2013;31:22.

46. Zang EA, Wynder EL. Differences in lung cancer risk between men and women: Examination of the evidence. J Natl Cancer Inst. 1996;88:183-92.

47. Shopland DR, Hartman AM, Gibson JT, Mueller MD, Kessler LG, Lynn WR. Cigarette smoking among U.S. adults by state and region: estimates from the current population survey. J Natl Cancer Inst. 1996;88:1748-58. 